\title{
Cochrane
}

Library

Cochrane Database of Systematic Reviews

\section{Early vitrectomy for exogenous endophthalmitis following surgery (Protocol)}

Muqit MMK, Mehat M, Bunce C, Bainbridge JW

Muqit MMK, Mehat M, Bunce C, Bainbridge JW.

Early vitrectomy for exogenous endophthalmitis following surgery (Protocol).

Cochrane Database of Systematic Reviews 2020, Issue 10. Art. No.: CD013760.

DOI: 10.1002/14651858.CD013760.

www.cochranelibrary.com

Early vitrectomy for exogenous endophthalmitis following surgery (Protocol) 
TABLE OF CONTENTS

HEADER 1

ABSTRACT

BACKGROUND

OBJECTIVES

METHODS

ACKNOWLEDGEMENTS

REFERENCES

APPENDICES

HISTORY

CONTRIBUTIONS OF AUTHORS

DECLARATIONS OF INTEREST

SOURCES OF SUPPORT 
[Intervention Protocol]

\section{Early vitrectomy for exogenous endophthalmitis following surgery}

Mahiul MK Muqit ${ }^{1}$, Manjit Mehat ${ }^{2}$, Catey Bunce ${ }^{3}$, James W Bainbridge ${ }^{2}$

1Moorfields Eye Hospital NHS Foundation Trust, London, UK. ${ }^{2}$ UCL Institute of Ophthalmology, London, UK. ${ }^{3}$ London, UK

Contact address: Mahiul MK Muqit, mahi.muqit1@nhs.net.

Editorial group: Cochrane Eyes and Vision Group.

Publication status and date: New, published in Issue 10, 2020.

Citation: Muqit MMK, Mehat M, Bunce C, Bainbridge JW. Early vitrectomy for exogenous endophthalmitis following surgery (Protocol). Cochrane Database of Systematic Reviews 2020, Issue 10. Art. No.: CD013760. DOI: 10.1002/14651858.CD013760.

Copyright @ 2020 The Cochrane Collaboration. Published by John Wiley \& Sons, Ltd.

\section{A B S T R A C T}

\section{Objectives}

This is a protocol for a Cochrane Review (intervention). The objectives are as follows:

To assess the potential role of combined pars plana vitrectomy and intravitreal antibiotics in the acute management of exogenous endophthalmitis, versus the standard of care, defined as vitreous tap and intravitreal antibiotics. 


\section{B A C K G R O U N D}

\section{Description of the condition}

Endophthalmitis is an inflammation of the internal eye tissues. It is a sight-threatening emergency that requires prompt diagnosis and treatment (Kresloff 1998). The condition is characterised by a purulent (containing pus) inflammation of the intraocular fluids. Endophthalmitis is broadly differentiated into exogenous or endogenous forms. In exogenous endophthalmitis, the infective agent is typically introduced into the eye through open globe trauma or intraocular surgeries, such as intravitreal injections, cataract, glaucoma, vitrectomy, or corneal surgery (Callegan 2007). In endogenous endophthalmitis, the infective agent is within the individual - for example in people with immunocompromised states. This is not the focus of this review. The incidence of exogenous endophthalmitis is variable, and dependent on the surgical intervention by which the infection was introduced. The incidence of endophthalmitis following intravitreal injection ranges from $0.033 \%$ to $0.082 \%$ (Clarke 2018). The post-cataract surgery endophthalmitis rate is between $0.06 \%$ and $0.2 \%$ ( Du 2014). The endophthalmitis incidence following pars plana vitrectomy (operations that remove the eye's internal jelly) is between 0.02 to $13 \%$ (Scott 2011). Although the frequency of endophthalmitis is relatively low, the volume of intraocular surgeries performed is expected to increase in response to the ageing global population.

\section{Description of the intervention}

Pars plana vitrectomy (PPV) is an established surgical technique for the removal of the vitreous cavity content. PPV surgery has changed significantly with recent advances in small gauge surgical instrumentation, wide-field viewing systems, and the availability of silicone oil for long-term retinal tamponade (the silicone oil prevents flow of fluid through the retinal break; (de Oliveira 2016)). There are recognised risks of PPV surgery that include infection, bleeding, loss of vision, eye pressure changes, macular oedema, cataract, retinal detachment, and suprachoroidal haemorrhage (Stein 2009). PPV is most commonly performed under local anaesthesia, with or without sedation; some people may require a general anaesthesia. PPV surgery may be performed for both diagnostic and therapeutic purposes, as either an emergency or planned procedure.

\section{How the intervention might work}

The current standard of care for suspected exogenous endophthalmitis is an urgent vitreous tap (biopsy), with an injection of intravitreal antibiotics at the time of clinical presentation. The response to treatment is then usually reassessed. Only in those people who experience continued clinical deterioration, is surgical intervention in the form of PPV performed. PPV has the advantage of clearing the visual axis, and promoting healing by removing the infected vitreous, which contains bacteria, bacterial endotoxins, immune cells, inflammatory cytokines, and other toxic mediators, which may incite retinal tissue damage (Forster 1980; Kuhn 2005). Failure to promptly remove these harmful factors, will result in continued exposure of the retina to toxins and pro-inflammatory products, even after the eye is sterilised by antibiotics (Astley 2016).

\section{Why it is important to do this review}

The early diagnosis and prompt treatment of endophthalmitis is critical in preventing irreversible eye damage and preserving visual function (Maguire 2008). Prompt delivery of intravitreal injection and sampling is well established as the standard of care for exogenous endophthalmitis (Kresloff 1998). However, the role of early PPV and intravitreal injection of antibiotics, defined as within one week of presentation, is less clearly understood, particularly in the context of non-cataract surgery-related endophthalmitis (Relhan 2018). A systematic review may help to ascertain whether early PPV intervention in exogenous endophthalmitis is beneficial, compared to the current standard of care.

\section{O B JECTIVES}

To assess the potential role of combined pars plana vitrectomy and intravitreal antibiotics in the acute management of exogenous endophthalmitis, versus the standard of care, defined as vitreous tap and intravitreal antibiotics.

\section{METHODS}

\section{Criteria for considering studies for this review}

\section{Types of studies}

We will include randomised controlled trials (RCTs) in this review.

\section{Types of participants}

We will include trials in which participants are defined as people who developed endophthalmitis within six weeks of any type of intraocular surgery or intervention, and have a best corrected visual acuity of worse than 30 letters, measured with an Early Treatment Diabetic Retinopathy Study (ETDRS) chart, at a starting distance of four metres. The types of surgery can include cataract surgery, glaucoma surgery, intravitreal injections, vitrectomy surgery, and corneal surgery. We will exclude participants with endogenous endophthalmitis.

\section{Types of interventions}

- Early intervention group. Early pars plana vitrectomy (PPV) surgery, with a second intravitreal antibiotic injection delivered within 48 hours for eyes that are non-responsive to the first intravitreal antibiotic dose.

- Comparator group. A second intravitreal antibiotic, 48 hours later, for eyes that are non-responsive to the first intravitreal antibiotic dose. Thereafter, the condition will be assessed on day five, and if the eye is non-responsive to the second intravitreal antibiotic dose, then further management will be actioned, according to standard of care hospital protocols, with a third intravitreal antibiotic dose.

\section{Types of outcome measures}

\section{Primary outcomes}

Proportion of people with a visual acuity (VA) improvement of 10 letters or more, measured with an ETDRS chart, at a starting distance of four metres, from randomisation to three months postrandomisation. 


\section{Secondary outcomes}

- Proportion of people with a VA improvement of 10 letters or more from randomisation to six months post-randomisation, measured with an ETDRS chart, at a starting distance of four metres

- Proportion of people requiring additional surgical procedures, including vitrectomy and cataract surgery, between randomisation and six months post-randomisation.

- Mean change in best-corrected visual acuity (BCVA), between randomisation and three months post-randomisation, measured with an ETDRS chart, at a starting distance of four metres.

- Mean change in BCVA, between randomisation and six months post-randomisation, measured with an ETDRS chart, at a starting distance of four metres

- Quality of life. Mean quality of life scores at six months postrandomisation, measured using a validated questionnaire.

\section{Adverse effects}

Any adverse effects identified during follow-up, in particular, retinal detachment, hypotony, intraocular haemorrhage, glaucoma,

\section{Search methods for identification of studies}

\section{Electronic searches}

The Cochrane Eyes and Vision Information Specialist will search the following electronic databases for randomised controlled trials and controlled clinical trials. There will be no restrictions to language or date of publication.

- Cochrane Central Register of Controlled Trials (CENTRAL; latest issue; which contains the Cochrane Eyes and Vision Trials Register) in the Cochrane Library (Appendix 1);

- MEDLINE Ovid (1946 to present; Appendix 2);

- Embase Ovid (1980 to present; Appendix 3);

- International Standard Randomised Controlled Trial Number registry (www.isrctn.com/editAdvancedSearch; Appendix 4);

- US National Institutes of Health Ongoing Trials Register ClinicalTrials.gov (www.clinicaltrials.gov; Appendix 5);

- World Health Organization International Clinical Trials Registry Platform (www.who.int/ictrp; Appendix 6).

\section{Searching other resources}

The lead author will scan the reference lists of the studies included in the review for information about further trials. We will not handsearch conference proceedings of journal specifically for the review.

\section{Data collection and analysis}

\section{Selection of studies}

We will only include randomised controlled trials that compare PPV with intravitreal injection of antibiotics, versus intravitreal injection of antibiotics alone, for the immediate management of exogenous endophthalmitis. Two review authors will independently carry out the study selection from the results of the searches (title and abstracts) to identify relevant studies. We will divide studies into 'definitely include', 'definitely exclude', and 'possibly include' categories, and resolve disagreements by discussion, or consultation (or both) with a third review author. In general, all citations considered not relevant at this stage will not be documented in the review, other than to note the number of these in a flow chart. We will obtain full-text copies of potentially relevant trials. We will make a final judgement regarding the inclusion or exclusion of studies in the 'possibly include' category after obtaining the full text of these articles. Two review authors will independently review all of the full-text reports; they will resolve disagreements by discussion, or consultation (or both) with a third review author. Review authors will not be masked to study authors, institutions, or journal and we will correspond with study authors to clarify study eligibility, as appropriate.

We will list all full texts excluded after examination in the excluded studies table, and provide a reason for exclusion.

For potentially eligible studies identified from trials registers, we will do the following:

- If the study has a completion date more than two years previously, we will look for publications of this trial, and contact the investigators if necessary to obtain published or unpublished data from the trial. If eligible, the study will be included in the review, regardless of whether we can identify a publication.

- If the study has a completion date within two years, or in the future, we will document the study in the ongoing studies section.

\section{Data extraction and management}

Two review authors will independently extract data, using a web-based online review management software (Covidence). We will ensure that the participants met our inclusion criteria for participants, described above. We will contact trial investigators for missing data. All data will be imported directly into Review Manager 5, and one author will check the accuracy of the data import (Review Manager 2020).

We will extract the following study characteristics from each included study:

1. Methods: method of allocation, masking (participant, provider, outcome), exclusions after randomisation, losses to follow-up, and compliance;

2. Participants: number randomised, age, sex, main inclusion and exclusion criteria;

3. Interventions: treatment, comparison intervention (control);

4. Outcomes: relevant outcomes for which data were collected in the trial and length of follow-up;

5. Notes: additional details relevant to that particular trial (e.g. funding sources).

See Appendix 7.

\section{Assessment of risk of bias in included studies}

Two review authors will independently assess, the risk of bias in each included study, using Cochrane's 'Risk of bias' tool (Higgins 2017).

We will resolve disagreements by discussion. 
We will specifically consider and report on the following parameters of bias:

1. Selection bias - random sequence generation, allocation concealment - was the sequence of allocation generated using a random procedure, and was the allocation concealed to people recruiting and enrolling participants, and to participants?

2. Performance bias - masking of participants and researchers - were the recipients of care unaware of their assigned intervention? Were the persons providing care unaware of the assigned intervention?

3. Detection bias - masking outcome assessors - were persons evaluating outcomes unaware of the assigned intervention?

4. Attrition bias - were the rates of follow-up and compliance similar in the groups? Was the analysis by intention-to-treat, and were there any post-randomisation exclusions?

5. Selective outcome reporting bias - is there any evidence that the outcomes that were measured were not reported?

We will classify each domain as low risk of bias, high risk of bias or unclear (lack of information or uncertainty of potential for bias). We will contact trial investigators for clarification of parameters graded as 'unclear'.

\section{Measures of treatment effect}

We will calculate the risk ratio for the following dichotomous outcomes:

- Proportion of people with a visual acuity (VA) improvement of 10 letters or more, measured with an ETDRS chart, at a starting distance of four metres, from randomisation to three months post-randomisation.

- Proportion of people with a visual acuity (VA) improvement of 10 letters or more, measured with an ETDRS chart, at a starting distance of four metres, from randomisation to six months postrandomisation.

We will compute odds ratios for the following outcomes (because these are rare, less than $10 \%)$ :

- proportion of people suffering harm during follow-up

- proportion of people requiring additional surgical procedures during follow-up

We will calculate the mean difference for the following continuous outcome

- mean change in best corrected visual acuity, between randomisation and three months post-randomisation, measured with an ETDRS chart, at a starting distance of four metres

- mean change in best corrected visual acuity between randomisation and six months post-randomisation, measured with an ETDRS chart, at a starting distance of four metres

- mean quality of life score at six months post-randomisation measuring using a validated questionnaire.

Where possible, we will check for the skewness of continuous data (Altman 1996).

\section{Unit of analysis issues}

We do not anticipate any unit of analysis issues with respect to eyes, because exogenous endophthalmitis usually only occurs in one eye, and therefore, people will be randomised to treatment, and one eye per person will be treated and reported.

\section{Dealing with missing data}

We will report the degree of missing data and check to see if reasons are provided by the authors on this. If similar rates and reasons are reported, and these rates are low, we may report the available case analysis only, but will comment upon this.

\section{Assessment of heterogeneity}

We will examine evidence of heterogeneity by reviewing the study characteristics and examining the forest plots. We will assess the inconsistency of effect estimates across studies using the $\mathrm{I}^{2}$ statistic, and the $\mathrm{Chi}^{2}$ test for heterogeneity. If the $\mathrm{I}^{2}$ statistic is greater than $50 \%$, we may consider this to be substantial heterogeneity. However, we will consider the magnitude and direction of effects in addition to $\mathrm{I}^{2}$.

\section{Assessment of reporting biases}

Should there be 10 trials or more included in a meta-analysis, we will construct funnel plots, and consider tests for asymmetry, to assess publication bias.

\section{Data synthesis}

If the $\mathrm{I}^{2}$ is greater than $50 \%$, and if there is significant clinical heterogeneity, we will not conduct a meta-analysis. Instead, we will present a tabulated or narrative summary, or both. If the $I^{2}$ is less than $50 \%$, and there is no clinical heterogeneity, we will combine the effect estimates in a meta-analysis, using a randomeffects model (provided we have three or more trials). We will use a fixed-effect model if there is no statistical or clinical heterogeneity, and if the number of trials is fewer than three. This is to avoid reporting less robust effect estimates that may result from randomeffects models in situations with very few trials. If $\mathrm{I}^{2}$ is greater than $50 \%$, but effect estimates are in the same direction, we may metaanalyse, but will stress the need for caution.

\section{Subgroup analysis and investigation of heterogeneity}

We do not intend to perform subgroup analysis.

\section{Sensitivity analysis}

We will perform sensitivity analyses to examine the impact of excluding studies at high risk of bias in any domain. We will examine whether the summary effect estimate is influenced by any assumptions that have been made during the review.

\section{Summary of findings}

We will prepare a 'Summary of findings' table presenting relative and absolute risks. Two authors will independently grade the overall quality of the evidence for each outcome using the GRADE classification GRADEpro GDT.

We will include these outcomes:

- Proportion of people with a visual acuity improvement of 10 letters or more at 3 months follow-up 
- Mean change in best-corrected visual acuity at 6 months followup

- Proportion of people requiring additional surgical procedures, including vitrectomy and cataract surgery, at any time during follow-up

- Mean quality of life scores at 6 months follow-up

- Proportion of people suffering adverse effects at any time during follow-up

\section{A C K N OWLEDGEMENTS}

The Methods section of this protocol is based on a standard template prepared by Cochrane Eyes and Vision Group (CEV). CEV will create and execute the electronic search strategies. We thank Felipe Dhawahir-Scala and Jennifer Evans for their comments on this protocol and Anupa Shah for her help with the editorial process. 


\section{REFERE N CES}

\section{Additional references}

\section{Altman 1996}

Altman DG, Bland JM. Detecting skewness from summary information. BMJ 1996;313(7066):1200.

\section{Astley 2016}

Astley RA, Coburn PS, Parkunan SM, Callegan MC. Modeling intraocular bacterial infections. Progress in Retinal and Eye Research 2016;54:30-48. [DOI: 10.1016/j.preteyeres.2016.04.007]

\section{Callegan 2007}

Callegan MC, Gilmore MS, Gregory M, Ramadan RT, Wiskur BJ, Moyer AL, et al. Bacterial endophthalmitis: therapeutic challenges and host-pathogen interactions. Progress in Retinal and Eye Research 2007;26(2):189-203.

\section{Clarke 2018}

Clarke B, Williamson TH, Gini G, Gupta B. Management of bacterial postoperative endophthalmitis and the role of vitrectomy. Surveys in Ophthalmology 2018;63(5):677-93.

\section{Covidence [Computer program]}

Veritas Health Innovation Covidence. Version accessed 20 July 2020. Melbourne, Australia: Veritas Health Innovation. Available at www.covidence.org.

\section{de Oliveira 2016}

de Oliveira PR, Berger AR, Chow DR. Vitreoretinal instruments: vitrectomy cutters, endoillumination and wide-angle viewing systems. International Journal of Retina and Vitreous 2016;2(28):1-15.

\section{Du 2014}

Du DT, Wagoner A, Barone SB, Zinderman CE, Kelman JA, MaCurdy TE, et al. Incidence of endophthalmitis after corneal transplant or cataract surgery in a medicare population. Ophthalmology 2014;121(1):290-8.

\section{Forster 1980}

Forster RK, Abbott RL, Gelender H. Management of infectious endophthalmitis. Ophthalmology 1980;87(4):313-9.

\section{GRADEpro GDT [Computer program]}

McMaster University (developed by Evidence Prime) GRADEpro GDT. Version accessed prior to 2 February 2020. Hamilton (ON):
McMaster University (developed by Evidence Prime). Available at gradepro.org.

\section{Higgins 2017}

Higgins JPT , Altman DG, Sterne JAC (editors). Chapter 8: Assessing risk of bias in included studies. In: Higgins JPT, Churchill R, Chandler J, Cumpston MS (editors), Cochrane Handbook for Systematic Reviews of Interventions version 5.2.0 (updated June 2017), Cochrane, 2017. Available from www.training.cochrane.org/handbook.

\section{Kresloff 1998}

Kresloff MS, Castellarin AA, Zarbin MA. Endophthalmitis. Survery of Ophthalmology 1998;43(3):193-224.

\section{Kuhn 2005}

Kuhn F, Gini G. Ten years after... are findings of the Endophthalmitis Vitrectomy Study still relevant today? Graefe's Archive for Clinical and Experimental Ophthalmology 2005;243(12):1197-9.

\section{Maguire 2008}

Maguire Jl. Postoperative endophthalmitis: optimal management and the role and timing of vitrectomy surgery. Eye 2008;22(10):1290-300

\section{Relhan 2018}

Relhan N, Forster RK, Flynn HW Jr. Endophthalmitis: then and now. American Journal of Ophthalmology 2018;187:xx-vii. [DOI: 10.1016/j.ajo.2017.11.021]

\section{Review Manager 2020 [Computer program]}

Review Manager (RevMan). Version 5.4.1. The Cochrane Collaboration, 2020.

\section{Scott 2011}

Scott IU, Flynn HW Jr, Acar N, Dev S, Shaikh S, Mittra RA, et al. Incidence of endophthalmitis after 20-gauge vs 23-gauge vs 25-gauge pars plana vitrectomy. Graefes Archive Clinical and Experimental Ophthalmology 2010;249(3):377-80.

\section{Stein 2009}

Stein JD, Zacks DN, Grossman D, Grabe H, Johnson MW, Sloan FA. Adverse events after pars plana vitrectomy among medicare beneficiaries. Archives of Ophthalmology 2009;127(12):1656-63.

\section{AP PE N DICES}

\section{Appendix 1. CENTRAL search strategy}

\#1 MeSH descriptor: [Vitrectomy] this term only \#2 PPV*

$\# 3$ vitrectom*

\#4 \#1 or \#2 or \#3

\#5 exogenous NEAR/3 endophthalmitis

\#6 \#4 and \#5 


\section{Appendix 2. MEDLINE Ovid search strategy}

1. randomized controlled trial.pt.

2. (randomized or randomised).ab,ti.

3. placebo.ab,ti.

4. dt.fs.

5. randomly.ab,ti.

6. trial.ab,ti.

7. groups.ab,ti.

8. or/1-7

9. exp animals/

10. exp humans/

11.9 not ( 9 and 10$)$

12. 8 not 11

13. vitrectomy/

14. PPV\$.tw.

15. vitrectom\$.tw.

16. or/13-15

17. (exogenous adj3 endophthalmitis).tw.

18. 16 and 17

19. 12 and 18

\section{Appendix 3. Embase Ovid search strategy}

1. exp randomized controlled trial/

2. exp randomization/

3. exp double blind procedure/

4. exp single blind procedure/

5. random\$.tw.

6. or/1-5

7. (animal or animal experiment).sh.

8. human.sh.

9. 7 and 8

10. 7 not 9

11.6 not 10

12. exp clinical trial/

13. (clin\$ adj3 trial\$).tw.

14. ((singl\$ or doubl\$ or trebl\$ or tripl\$) adj3 (blind\$ or mask\$)).tw.

15. exp placebo/

16. placebo\$.tw.

17. random $\$$.tw.

18. exp experimental design/

19. exp crossover procedure/

20. exp control group/

21. exp latin square design/

22. or/12-21

23. 22 not 10

24. 23 not 11

25. exp comparative study/

26. exp evaluation/

27. exp prospective study/

28. (control\$ or prospectiv\$ or volunteer\$).tw.

29. or $/ 25-28$

30.29 not 10

31.30 not (11 or 23$)$

32. 11 or 24 or 31

33. vitrectomy/

34. PPV\$.tw.

35. vitrectom\$.tw.

36. or/33-35

37. (exogenous adj3 endophthalmitis).tw.

38. 36 and 37

39. 32 and 38 


\section{Appendix 4. ISRCTN search strategy}

(vitrectomy OR PPV) AND (exogenous endophthalmitis)

\section{Appendix 5. ClinicalTrials.gov search strategy}

(vitrectomy OR PPV) AND (exogenous endophthalmitis)

\section{Appendix 6. WHO ICTRP search strategy}

vitrectomy AND exogenous endophthalmitis OR PPV AND exogenous endophthalmitis

\section{Appendix 7. Data on study characteristics}

\begin{tabular}{|c|c|c|}
\hline Mandatory items & Details & Optional items \\
\hline \multicolumn{3}{|l|}{ Methods } \\
\hline Study design & $\begin{array}{l}\text { - Parallell group } \mathbf{R C T} \text { i.e. people randomised to treatment } \\
\text { - Within-person } \mathbf{R C T} \text { i.e. eyes randomised to treatment } \\
\text { - Cluster-RCT i.e. communities randomised to treatment } \\
\text { - Cross-over } \mathbf{R C T} \\
\text { - Other, specify }\end{array}$ & $\begin{array}{l}\text { Exclusions after randomisa- } \\
\text { tion } \\
\text { Losses to follow-up } \\
\text { Number ran- } \\
\text { domised/analysed } \\
\text { How were missing data han- } \\
\text { dled? e.g. available case } \\
\text { analysis, imputation meth- } \\
\text { ods } \\
\text { Reported power calculation } \\
\text { (Y/N), if yes, sample size and } \\
\text { power } \\
\text { Unusual study design/is- } \\
\text { sues }\end{array}$ \\
\hline
\end{tabular}

Eyes or Unit of randomisation/ unit of analysis

\begin{abstract}
- One eye included in study, specify how eye selected
- Two eyes included in study, both eyes received same treatment, briefly specify how analysed (best/worst/average/both and adjusted for within person correlation/both and not adjusted for within person correlation) and specify if mixture one eye and two eye
\end{abstract}

- Two eyes included in study, eyes received different treatments, specify if correct pair-matched analysis done

\section{Participants}

Country

\section{Setting}

Ethnic group

Equivalence of baseline

characteristics $(\mathrm{Y} / \mathrm{N})$

\footnotetext{
Total number of participants

This information should be collected for total study population recruited into the study. If these data are only reported for the people who were followed up, please indicate.
}

\section{Number (\%) of men and} women 
(Continued)

Average age and age range

Inclusion criteria

Exclusion criteria

\section{Interventions}

\begin{tabular}{ll}
\hline Intervention $(\mathrm{N}=)$ & $\cdot$ Number of people randomised to this group \\
Comparator $(\mathrm{N}=)$ & $\cdot$ Drug (or intervention) name \\
See MECIR 66 & $\cdot$ Dose \\
& $\cdot$ Frequency \\
& $\cdot$ Route of administration
\end{tabular}

\section{Outcomes}

Primary and secondary outcomes as defined in study reports

List outcomes

Adverse events reported $(\mathrm{Y} / \mathrm{N})$

Length of follow-up and intervals at which outcomes assessed
Planned and actual length

of follow-up

\section{See MECIR R67}

\section{Notes}

Date conducted

Specify dates of recruitment of participants $\mathrm{mm} / \mathrm{yr}$ to $\mathrm{mm} / \mathrm{yr}$

Full study name: (if applicable)

Reported subgroup analy$\operatorname{ses}(\mathrm{Y} / \mathrm{N})$

Were trial investigators contacted?

\section{Sources of funding See \\ MECIR 69}

Declaration of interest

\section{See MECIR 70}

Included in trials registry $\quad \mathrm{Y} / \mathrm{N}$; include registration number if available

\section{H IST O R Y}

Protocol first published: Issue 10, 2020

\section{CONTRIBUTIONS OF AUTHORS}

Conceiving the review idea: MMKM

All authors were involved in writing drafts of the protocol.

\section{DECLARATIONS OF INTEREST}

MMKM is a Consultant on a Scientific Advisory Board for Pixium Vision. This is an unpaid role MM: no conflicts of interest to declare 
$\mathrm{CB}$ is a co-applicant on a study investigating early vitrectomy in endophthalmitis

JB has received payment for consultancy work from MeiraGTx Ltd and Novartis. Neither company has a direct interest in the subject of this review

\section{SOURCES OF SUPPORT}

\section{Internal sources}

- No sources of support supplied

\section{External sources}

- National Institute for Health Research (NIHR), UK

* Richard Wormald, Co-ordinating Editor for Cochrane Eyes and Vision (CEV) acknowledges financial support for his CEV research sessions from the Department of Health through the award made by the National Institute for Health Research to Moorfields Eye Hospital NHS Foundation Trust and UCL Institute of Ophthalmology for a Specialist Biomedical Research Centre for Ophthalmology.

* This protocol was supported by the NIHR, via Cochrane Infrastructure funding to the CEV UK editorial base.

The views and opinions expressed therein are those of the authors and do not necessarily reflect those of the Systematic Reviews Programme, NIHR, NHS or the Department of Health. 